Derivations usually proceed through formal transformations followed by a brief physical synopsis of the results. 'The numerous insights are more mathematical than physical.

This is a sedate book. Little remains of Robertson's flamboyant style-his caustic comment, his witty barb. Only on page 60 are we allowed a brief glimpse, and even this is "censored". The man who, in his milder moments, called Einstein's cosmological constant a "spook in the ether" and the substratum a "basic undergarment" 1 is hardly represented here. But Robertson's science, his distinctive style of relativity, is represented very well.

William C. Saslaw

${ }^{1}$ Path. Astron. Soc. Pacific, 67, 82 (1955).

\section{MATHS FOR BIOLOGISTS}

\section{Biomathematics}

The Principles of Mathematics for Students of Biological and General Science. By Cedric A. B. Smith. Vol. 1: Algebra, Geometry, Calculus. Fourth edition. Pp. xii + 523. (1965.) 63s. Vol. 2: Numerical Methods, Matrices, Probability and Statistics. Fourth edition. Pp. $\mathrm{x}+682$. (1969.) 138s. (Charles Griffin: London.)

THE first edition of Biomathematics was written by the physician W. M. Feldman in 1923 to explain the elements of mathernatics, in particular the differential and integral calculus, to research workers in the biological and medical sciences. The success of Feldman's book, of which a second edition appeared in 1935, was due to its simple, non-rigorous style and to the wealth of practical examples which demonstrated to the scientist the value of formulating biological problems in mathematical terms. The third edition of Biomathematics was written by C. A. B. Smith and appeared in 1954. This was in fact an entirely new book, covering rather more ground than the original editions at considerably greater length. In the fourth edition, which has been divided into two volumes, Smith has left almost unchanged the chapters on algebra, geometry and calculus in the first volume but has greatly expanded the coverage of matrices and of probability and statistics which now occupy the whole of the second volume.

In the first volume of the present edition Smith has written a very useful introduction to the calculus for biologists with little previous knowledge of mathematics. The level of sophistication is rather higher than in Feldman's edition, and the temptation to sacrifico simplicity for mathematical elegance has not always been resisted (for example, in the definition of the logarithm by means of the equiangular spiral), but any biologist who is prepared to read this volume carefully should find it both intelligible and interesting. I am doubtful, however, whether the same reader will gain as much from the second volume in which the author develops his own rather idiosyncratic views on statistical inference based on the use of "pignic" (or betting) odds. The reader who is unfamiliar with, or uninterested in, the current argument about tho foundations of statistical inference is unlikely to find this volume good value for money, but there is a great deal in it to interest the statistician.

M. G. Bulmer

\section{ELECTRODE PROCESSES}

\section{Electrochemistry at Solid Electrodes}

By Ralph N. Adams. (Monographs in Electroanalytical Chemistry and Electrochomistry.) Pp. xiii +402 . (Dekker: New York, January 1969.) \$18.75.

THIS is a good book, but the prospective reader should be warned against being misled by the title. "Electro- chemistry at Solid Electrodes" could imply a large part of the whole subject of electrochemistry. In reality, the book has a much smaller range; it is primarily concerned with irreversible electrode processes and their investigation by some techniques that have been developed in recent years.

The first six chapters, which occupy nearly half the book, describe these techniques; they might be broadly summarized as voltammetry at controlled potential, chronopotentiometry and rotating disk electrodes, although the contents really cover a rather wider field. The principles are clearly explained, and there is a substantial amount on convective transport. But alternating current techniques (Faradaic and Warburg impedances) are not discussed.

The second half of the book deals with applications to the study of clectrode processes, including the evaluation of diffusion coefficients and of kinetic parameters. A section of chapter eight stresses the importance of supplementing purely electrochemical studics of reaction mechanisms by other techniques; the applications of EPR are described.

The final chapter is devoted to the electrode reactions of organic compounds-especially oxidation. This is a subject in which there is currently much interest and in which the dropping mercury electrode often cannot compete with chemically inert solid electrodes. It opens with the statement that "The main purpose of all the previous sections in this monograph is to provide background for a survey and discussion of electrochemical reactions of organic materials at solid electrodes". I feel that this undervalues the earlier parts of the book, but the chapter in question certainly provides an authoritative account of the subject.

The book contains a great deal of information on practical matters, such as construction of electrodes; this information, in conjunction with the discussion of general theoretical principles to be found in the earlier chapters, will be particularly useful to anyone wishing to start work in the field.

J. N. AGAR

\section{RARE DISORDER}

\section{Anorexia Nervosa}

By Peter Dally. Pp. ix +137 . (Heinemann (Medical): London, May 1969.) $30 s$.

THIS monograph is largely a report by the author of $h$ is extensive clinical experience with anorexia nervosa, a condition largely occurring in young women, and characterized by amenorrhoea, weight loss, malnutrition, accompanied by psychopathological factors. For several years he has been systematically collecting information on a group of such patients which now numbers one hundred and forty. In his report, the author attempts to bring some order to these data by identifying subgroups of patients with differing personalities which he claims arc sometimes associated with difforing basic attitudes within the illness and different outcomes.

In the past, there have been many insubstantial hypotheses concerning the aetiology and nature of anorexia nervosa, often based on experience with just a few patients. This situation is not surprising when the rarity of the condition and the complex clinical condition of these often seriously ill, pathotic, reluctant patients with their so specific and metabolic and behavioural disorders are considered. At last, reports based on wider experience such as the one under review are beginning to appear.

The author has used just sufficient space in his short introductory chapters to acquaint the reader clearly with the historical background. In his fourth chapter he launches into his attempted classification of his own series of patients into a large obsessional $(O)$ group containing half the total patients, and smaller hysterical $(H)$ and mixed $(M)$ groups. It is at this stage, when he is attexopting to bo most orderly, that $I$ find him most ambiguous. 